\title{
A HYPERBOLIC SYSTEM AND THE COST OF THE NULL CONTROLLABILITY FOR THE STOKES SYSTEM
}

\author{
F. W. CHAVES-SILVA
}

\begin{abstract}
This paper is devoted to study the cost of the null controllability for the Stokes system. Using the control transmutation method we show that the cost of driving the Stokes system to rest at time $T$ is of order $e^{C / T}$, as in the case of the heat equation. For this to be possible, we are led to study the exact controllability of one hyperbolic system with a resistance term, which will be done under assumptions on the control region.
\end{abstract}

\section{INTRODUCTION}

Let $\Omega \subset \mathbb{R}^{N}(N \geq 1)$ be a bounded connected open set, whose boundary $\partial \Omega$ is regular enough. Let $T>0$ and let $\omega$ be a nonempty subsets of $\Omega$ which will usually be referred to as a control domain. We will use the notation $Q=\Omega \times(0, T)$ and $\Sigma=\partial \Omega \times(0, T)$ and we will denote by $\nu(x)$ the outward normal to $\Omega$ at the point $x \in \partial \Omega$.

Given $u_{0} \in L^{2}(\Omega)$, it is well-known (see [12], [13]) that there exists $f \in L^{2}(\omega \times(0, T))$ such that the associated solution $v$ to the heat equation

$$
\mid \begin{array}{ll}
v_{t}-\Delta v=f 1_{\omega} & \text { in } Q \\
v=0 & \text { on } \Sigma \\
v(0)=v_{0} & \text { in } \Omega
\end{array}
$$

satisfies:

$$
v(T)=0 .
$$

In other words, the heat equation is null controllable for any control domain and any initial data $v_{0} \in L^{2}(\Omega)$. Moreover, one also has the following estimate:

$$
\left\|f 1_{\omega}\right\|_{L^{2}(Q)} \leq C_{h}\left\|v_{0}\right\|_{L^{2}(\Omega)},
$$

for a constant $C_{h}$, the cost of controllability for the heat equation, of the form $e^{C(\Omega, \omega)(1+1 / T)}$, i.e., the heat equation has a cost of controllability of order $e^{C / T}$.

As pointed out in [6] (see also [7], [21], [22], [27]), the main reason for the form of the constant $C_{h}$ in (1.3) is due to the fact that the fundamental solution of the heat equation in $\mathbb{R}^{N}$ is given by

$$
\Phi(x, t)=\frac{1}{(4 \pi t)^{N / 2}} e^{-\frac{|x|^{2}}{4 t}}
$$

Key words and phrases. Stokes system; null controllability; cost of control; hyperbolic system; pressure term. 
As in the case of the heat equation, if one now considers the Stokes system

$$
\begin{array}{ll}
y_{t}-\Delta y+\nabla p=g 1_{\omega} & \text { in } Q, \\
\text { div } y=0 & \text { in } Q, \\
y=0 & \text { on } \Sigma, \\
y(0)=y_{0} & \text { in } \Omega,
\end{array}
$$

it is also well-known (see, for instance, [9]) that, given $y_{0} \in L^{2}(\Omega)$ with div $y_{0}=0$, there exists $g \in L^{2}(\omega \times(0, T))$ such that the associated solution $y_{0}$ to $(1.5)$ satisfies:

$$
y(T)=0 .
$$

Nevertheless, unlike the case of the heat equation, for the Stokes system, the known results in the literature (for instance [9]) gives

$$
\left\|g 1_{\omega}\right\|_{L^{2}(Q)} \leq C_{S}\left\|y_{0}\right\|_{L^{2}(\Omega)},
$$

for a constant $C_{S}$, the cost of controllability for the Stokes equation, of the form $e^{C(\Omega, \omega)\left(1+1 / T^{4}\right)}$, i.e., a cost of order $e^{C / T^{4}}$.

Since the fundamental solutions of the heat and the Stokes system have, at least for $N=2,3$, the same behavior in time (see [16], [17], [25]), looking to (1.3) and (1.6), the following natural question arises:

Question 1.1. Do the cost of the controllability for the heat equation and the Stokes system have the same order in time?

When trying to answer Question 1.1, the first attempt is to analyze the different ways one can prove (1.3) and (1.6). In fact, there exist at least two different ways one can prove (1.3), the first one is based on spectral decompositions, the so-called Lebeau-Robbiano strategy (see [18]), the second is based on the use of Carleman inequalities (see [12], [13]). For the Stokes system, it seems that a Lebeau-Robbiano strategy is very difficult to prove, since one must deal with the pressure, and the most known method used to prove (1.6) is based on Carleman inequalities (see [9]).

The main difference when proving (1.3) and (1.6) by mean of Carleman inequalities are the weights one must use. Indeed, for the heat equation the weights used are of the form

$$
\rho(t)=\frac{e^{C /(t(T-t))}}{t(T-t)},
$$

while for the Stokes system the weights are of the form

$$
\rho(t)=\frac{e^{C /\left(t^{4}(T-t)^{4}\right)}}{t^{4}(T-t)^{4}} .
$$

The reason why one has different weights for the Stokes system than for the heat equation is due to the fact that one must deal with the pressure term in the first equation. If we were able to use weights like (1.7) for the Stokes system then these two equations would have costs of controllability of same order. However, a careful analysis of both proofs indicates that this is not the case, but also gives hope, since the obstruction one has when dealing with the pressure seems to be just technical. 
The main objective of this paper is to show that heat and the Stokes system have costs of controllability of same order. Our strategy will not be based on the use of Carleman inequalities but rather on the application of the Control Transmutation Method, shortly CTM. This method is based on the idea that when faced with a new problem, one good strategy is try to reduce it to a previously solved problem, or at least to a simpler one.

In order to use the CTM, we are led to study the null controllability of the following hyperbolic system with a pressure term:

$$
\mid \begin{array}{ll}
u^{\prime \prime}-\Delta u+\nabla p=h 1_{\omega} & \text { in } Q, \\
d i v u=0 & \text { in } Q, \\
u=0 & \text { on } \Sigma, \\
u(0)=u^{0}, u^{\prime}(0)=u^{1} & \text { in } \Omega .
\end{array}
$$

The idea then is as follows. If one can show that system (1.9) is null controllable, then the CTM can be applied in order to guarantee the null controllability for the Stokes system (1.5). Moreover, if one knows the cost of controlling (1.9) then the cost of the controllability for (1.5) is also known (see Theorem 4.2).

Concerning the controllability of (1.9), as far as we know, the only result available in the literature is [24]. In her thesis, the author shows the exact controllability of (1.9) when the control is acting on a part of the boundary. However, it seems that no controllability results are known when the control is acting internally, i.e., acting on a part of the domain. The main reason for this seems to be the fact that system (1.9) is not of Cauchy-Kowalewski type, which makes impossible the use of Holgrem's Theorem as in the case of the wave equation.

This paper is organized as follows. In section 2 we prove the internal null controllability of (1.9), under geometrical restrictions on the control domain. In section 5, using the CTM, we prove that system (1.5) has the same cost of controllability if the initial data is regular enough. Finally, in section 6 , we prove that we can take initial data less regular and still have the same order of controllability for the Stokes system as for the heat equation.

\section{A Hyperbolic System With a Pressure term}

This section is devoted to prove a null controllability result for the hyperbolic system (1.9). This result will allow us to show, in the next section, the null controllability of the Stokes system (1.5) and also get a precise estimate on the cost of this controllability.

During this paper, we assume that $\Omega$ is star-shaped with respect to the origin, i.e., there exists $\gamma>0$ such that

$$
x \cdot \nu(x) \geq \gamma>0 \text { on } \forall x \in \partial \Omega .
$$

Given a point $x_{0} \in \mathbb{R}^{N}$, we divide the boundary $\partial \Omega$ into two pieces

$$
\partial \Omega_{0}=\{x \in \partial \Omega ; m(x) \cdot \nu>0\} \text { and } \partial \Omega_{*}=\partial \Omega \backslash \partial \Omega_{0},
$$

where $m(x)=x-x_{0}$. We also define

$$
R\left(x_{0}\right)=\max _{x \in \bar{\Omega}}|m(x)| .
$$

Our control region $\omega$ will be a nonempty subset of $\Omega$ satisfying:

$$
\exists \mathcal{O} \subset \mathbb{R}^{N}, \mathcal{O} \text { is a neighborhood of } \overline{\partial \Omega_{0}} \text { and } \omega=\Omega \cap \mathcal{O} .
$$


We introduce

$$
\mathcal{V}=\left\{v \in C_{0}^{\infty}(\Omega) ; \operatorname{div} v=0\right\}
$$

and consider the following Banach spaces (provided with the topology of $H_{0}^{1}(\Omega)^{N}, L^{2}(\Omega)^{N}$, $L^{2}(\Sigma)^{N}$ and $H^{-1}(\Omega)^{N}$, respectively):

$$
\begin{gathered}
V=\left\{u \in H_{0}^{1}(\Omega)^{N} ; \operatorname{div} u=0\right\}, \\
H=\left\{u \in L^{2}(\Omega)^{N} ; \operatorname{div} u=0, u \cdot \nu=0 \text { on } \partial \Omega\right\}, \\
Z=\left\{v \in L^{2}(\Sigma)^{N} ; \iint_{\Sigma} v \cdot \nu d \Sigma=0\right\}, \\
W=\text { closure of } \mathcal{V} \text { in } H^{-1}(\Omega)^{N} .
\end{gathered}
$$

Note that

$$
W \subset\left\{f \in H^{-1}(\Omega)^{N} ; \operatorname{div} f=0 \text { in } \mathcal{D}^{\prime}(\Omega)\right\} .
$$

Given $u^{0} \in V$ and $u^{1} \in H$, we want to find $h$ such that the solution $u$ of

satisfies:

$$
\mid \begin{array}{ll}
u^{\prime \prime}-\Delta u+\nabla p=h 1_{\omega} & \text { in } Q, \\
\operatorname{div} u=0 & \text { in } Q, \\
u=0 & \text { on } \Sigma, \\
u(0)=u^{0}, u^{\prime}(0)=u^{1} & \text { in } \Omega
\end{array}
$$

$$
u(T)=u^{\prime}(T)=0 .
$$

Systems like (2.3) are simple models of dynamical elasticity for incompressible materials. They also appear in coupled elasto-thermicity problems where one of the coupling parameter (related to compressibility properties) tends to infinity (see [20]).

Concerning the existence and uniqueness of solution to (2.3), we have:

Theorem 2.1. Let $\left(u^{0}, u^{1}, h\right) \in V \times H \times L^{1}(0, T ; H)$. Then there exists a unique weak solution $u$ of the problem (2.3) such that

$$
u \in C([0, T] ; V) \cap C^{1}([0, T] ; H)
$$

and $u$ satisfies:

$$
\frac{1}{2}\left|u^{\prime}(t)\right|_{H}^{2}+\frac{1}{2}\|u(t)\|_{V}^{2}=\frac{1}{2}\left|u^{1}\right|_{H}^{2}+\frac{1}{2}\left\|u^{0}\right\|_{V}^{2}+\int_{0}^{t}\left(h(s), u^{\prime}(s)\right)_{H} d s, \quad \forall t \in[0, T] .
$$

Moreover, the linear mapping

$$
\begin{gathered}
V \times H \times L^{1}(0, T ; H) \longrightarrow C([0, T] ; V) \cap C^{1}([0, T] ; H) \\
\left(u^{0}, u^{1}, f\right) \mapsto u
\end{gathered}
$$

is continuous.

Proof. Since the proof is performed using the standard Galerkin method and energy inequalities, it will not be reproduced here. 
Now, we focus on the null controllability problem for (2.3). As we will see (Theorem 4.2), the null controllability for (2.3) is equivalent to a observability inequality its adjoint system

$$
\mid \begin{array}{ll}
\phi^{\prime \prime}-\Delta \phi+\nabla q=0 & \text { in } Q, \\
\operatorname{div} \phi=0 & \text { in } Q, \\
\phi=0 & \text { on } \Sigma, \\
\phi(0)=\phi^{0}, \phi^{\prime}(0)=\phi^{1} & \text { in } \Omega,
\end{array}
$$

where $\phi^{0} \in H$ and $\phi^{1} \in W$.

In other words, to prove the null controllability of (2.3), it is sufficient to prove the existence of a positive constant $C$ such that

$$
\left|\phi^{0}\right|_{H}^{2}+\left\|\phi^{1}\right\|_{W}^{2} \leq C \iint_{\omega \times(0, T)}|\phi|^{2} d x d t
$$

for all solutions of (2.4).

Remark 2.2. The solution $\phi$ of (2.4) is defined by transposition. More precisely, $\phi \in L^{2}(0, T ; H)$ is an ultra weak solution of (2.4) if

$$
\int_{0}^{T}(\phi(t), h(t))_{H} d t=<\phi^{1}, u(0)>_{H^{-1}(\Omega), H_{0}^{1}(\Omega)}-\left(\phi^{0}, u^{\prime}(0)\right)_{H},
$$

for all $h \in L^{2}(0, T: H)$, where $u$ is the weak solution of

$$
\mid \begin{array}{ll}
u^{\prime \prime}-\Delta u+\nabla p=h & \text { in } Q, \\
\operatorname{div} u=0 & \text { in } Q, \\
u=0 & \text { on } \Sigma, \\
u(T)=0, u^{\prime}(T)=0 & \text { in } \Omega .
\end{array}
$$

As in the case of the wave equation, in order to show an observability inequality with internal observation for (2.4), first we show a boundary observability inequality for this system. The result is as follows.

Theorem 2.3. If we take $T>2 R\left(x_{0}\right)$ then, for every solution of (2.4) with initial data $\left(\phi^{0}, \phi^{1}\right) \in$ $V \times H$, the following estimates holds:

$$
\left|\phi^{1}\right|_{H}^{2}+\left\|\phi^{0}\right\|_{V}^{2} \leq \frac{R\left(x_{0}\right)}{2\left(T-2 R\left(x_{0}\right)\right)} \iint_{\Sigma_{0}}\left(\frac{\partial \phi}{\partial \nu}\right)^{2} d \Sigma .
$$

Proof. Using Lemma A.1 in Appendix, with $\bar{q}$ being the vector field $m(x)=x-x_{0}$, we have:

$$
\frac{1}{2} \iint_{\Sigma} m \cdot \nu\left(\frac{\partial \phi}{\partial \nu}\right)^{2} d \Sigma=\left.\left(\phi^{\prime}(t), m(x) \nabla \phi(t)\right)\right|_{0} ^{T}+\iint_{Q}|\nabla \phi|^{2} d x d t+\frac{n}{2} \iint_{Q}\left(\left|\phi^{\prime}\right|^{2}-|\nabla \phi|^{2}\right) d x d t .
$$

Next, multiplying $(2.4)_{1}$ by $\phi$ and integrate by parts, we easily see that

$$
\left.\left(\phi^{\prime}(t), \phi(t)\right)\right|_{0} ^{T}=\iint_{Q}\left|\phi^{\prime}\right|^{2} d x d t-\iint_{Q}|\nabla \phi|^{2} d x d t .
$$

Then, using this last identity and the fact that

$$
\left|\phi^{\prime}(t)\right|_{H}^{2}+\|\phi(t)\|_{V}^{2}=\left|\phi^{1}\right|_{H}^{2}+\left\|\phi^{0}\right\|_{V}^{2} \quad \forall t \in[0, T],
$$


we obtain

$$
\left.\left(\phi^{\prime}(t), m \nabla u(t)+\frac{n-1}{2} u(t)\right)\right|_{0} ^{T}+T\left(\left|\phi^{1}\right|_{H}^{2}+\left\|\phi^{0}\right\|_{V}^{2}\right)=\frac{1}{2} \iint_{\Sigma} m \cdot \nu\left(\frac{\partial \phi}{\partial \nu}\right)^{2} d \Sigma .
$$

We also have

$$
\left|m \nabla u(t)+\frac{n-1}{2} u(t)\right|^{2} \leq R\left(x_{0}\right)|\nabla \phi(t)|^{2} \quad \forall t \in[0, T],
$$

wich implies, by Gronwall inequality,

$$
\left|\left(\phi^{\prime}(t), m \nabla \phi+\frac{n-1}{2} \phi\right)_{0}^{T}\right| \leq 2 R\left(x_{0}\right)\left(\left|\phi^{1}\right|_{H}^{2}+\left\|\phi^{0}\right\|_{V}^{2}\right) .
$$

Finally, combining all the above estimates, we conclude that

$$
\left(T-2 R\left(x_{0}\right)\right)\left(\left|\phi^{1}\right|_{H}^{2}+\left\|\phi^{0}\right\|_{V}^{2}\right) \leq \frac{R\left(x_{0}\right)}{2} \iint_{\Sigma_{0}}\left(\frac{\partial \phi}{\partial \nu}\right)^{2} d \Sigma
$$

and the result follows.

Concerning Theorem 2.3, it is important to mention that this result was already obtained in [24]. Nevertheless, in this paper we go a step further and use this result in order to show the internal observability inequality (2.5).

Now we prove a very interesting result, which says that a proof of (2.5) can be obtained if one is able to prove a special kind of observability inequality for system (2.4).

Theorem 2.4. Assume that there exists a constant $C>0$ such that, for every $\left(\phi^{0}, \phi^{1}\right) \in V \times H$, the weak solution $\phi$ of (2.4) satisfies

$$
\left\|\phi^{0}\right\|_{V}^{2}+\left|\phi^{1}\right|_{H}^{2} \leq C \iint_{\omega \times(0, T)}\left|\phi^{\prime}\right|^{2} d x d t
$$

then inequality (2.5) holds for all solutions of (2.4) with initial data $\left(\phi^{0}, \phi^{1}\right)$ in $H \times W$.

Proof. In fact, given $\left(\phi^{0}, \phi^{1}\right) \in H \times W$, we take $\xi \in V$ such that $\xi$ solution, together with some $\pi \in L_{0}^{2}(\Omega)$, of $-\Delta \xi+\nabla \pi=\phi^{1}$. We define

$$
\psi(x, t)=-\xi(x)+\int_{0}^{t} \phi(x, s) d s,
$$

where $\phi=\phi(x, t)$ is the solution of (2.4) associated to the initial data $\left(\phi^{0}, \phi^{1}\right)$.

Integrating system (2.4) in time, we obtain

$$
\phi(t)^{\prime}-\phi^{\prime}(0)-\Delta \int_{0}^{t} \phi(x, s) d s+\nabla\left(\int_{0}^{t} q(x, s) d s\right)=0
$$

and it follows that

$$
\psi^{\prime \prime}(x, t)-\phi^{1}-\Delta(\psi(x, t)+\xi(x))+\nabla\left(\int_{0}^{t} q(x, s) d s\right)=0 .
$$


Using the definition of $\xi$, we see that $\psi$ solves the following hyperbolic system:

$$
\mid \begin{array}{ll}
\psi^{\prime \prime}-\Delta \psi+\nabla \hat{q}=0 & \text { in } Q, \\
\operatorname{div} \psi=0 & \text { in } Q, \\
\psi=0 & \text { on } \Sigma, \\
\psi(0)=-\xi, \psi^{\prime}(0)=\phi^{0} & \text { in } \Omega .
\end{array}
$$

Now, if inequality (2.7) is true, using energy inequality for (2.8), we have

$$
\|\xi\|_{V}^{2}+\left|\phi^{0}\right|_{H}^{2} \leq C \iint_{\omega \times(0, T)}|\phi|^{2} d x d t .
$$

The proof is then finished observing that (2.9) implies (2.5).

\section{A FIRST OBSERVABILITY INEQUALITY}

In this section we prove a variant of the observability inequality (2.7), which will be useful for our purposes in the next section. Indeed, we prove the following result.

Proposition 3.1. Assume $\omega$ satisfies (2.2) and let $T>2 R\left(x_{0}\right)$. Then, there exists a constant $C>0$ such that, for every $\left(\phi^{0}, \phi^{1}\right) \in V \times H$, the weak solution $\phi$ of (2.4) satisfies:

$$
\|\xi\|_{V}^{2}+\left|\phi^{0}\right|_{H}^{2} \leq C \iint_{\omega \times(0, T)}\left(\left|\phi^{\prime}\right|^{2}+|\phi|^{2}\right) d x d t .
$$

Before proving Proposition 3.1, we first prove the following lemma.

Lemma 3.2. Let $m \in C^{1}(\bar{\Omega})^{N}$. Then, for all regular solutions of (2.4), the following identity holds

$$
\langle\nabla q, m \cdot \nabla \phi\rangle_{L^{2}(Q)}=-\langle\nabla q, \phi \cdot \nabla m\rangle_{L^{2}(Q)}+\langle\nabla q, \phi(\operatorname{div} m)\rangle_{L^{2}(Q)^{N}} .
$$

Proof. Let us set

$$
X=-\iint_{Q} \frac{\partial q}{\partial x_{i}} m_{k} \frac{\partial \phi^{i}}{\partial x_{k}} d x d t
$$

Integrating by parts with respect to $x_{k}$, and using the fact that $\phi=0$ on $\Sigma$, we get

$$
X=\iint_{Q} \frac{\partial}{\partial x_{k}}\left(\frac{\partial q}{\partial x_{i}} m_{k}\right) \phi^{i} d x d t=\iint_{Q} \frac{\partial^{2} p}{\partial x_{k} \partial x_{i}} m_{k} \phi^{i} d x d t+\iint_{Q} \frac{\partial q}{\partial x_{i}} \frac{\partial m_{k}}{\partial x_{k}} \phi^{i} d x d t .
$$

We integrate by parts again the first integral, this time with respect to $x_{i}$, we obtain

$$
\iint_{Q} \frac{\partial q}{\partial x_{k}} \frac{\partial}{\partial x_{i}}\left(m_{k} \phi^{i}\right) d x d t=-\iint_{Q} \nabla p \phi \cdot \nabla m d x d t
$$

Hence, we conclude that

$$
X=-\iint_{Q} \nabla q \phi \cdot \nabla m d x d t+\iint_{Q} \nabla q \phi(\operatorname{div} m) d x d t .
$$

Let us now prove Proposition 3.1. 
Proof of Proposition 3.1. We will use the following notation:

$$
E(t)=\left|\phi^{\prime}(t)\right|_{H}^{2}+\|\phi(t)\|_{V}^{2}, \quad \forall t \in[0, T] .
$$

Let us consider first the case where $\phi$ is regular, for instance, we can take $\phi^{0} \in V \cap H^{4}(\Omega)$ and $\phi^{1} \in V \cap H^{2}(\Omega)$.

Using the change of variables $T \tau=(T-2 \epsilon) t+T \epsilon$, wich implies $\epsilon \leq \tau \leq T-\epsilon$, we rewrite the inequality (2.6) in Theorem 2.3 as

$$
E(0) \leq C \int_{\epsilon}^{T-\epsilon} \int_{\Gamma_{0}}\left(\frac{\partial \phi}{\partial \nu}\right)^{2} d \Sigma .
$$

Next, we consider a vector field $h \in C^{2}(\bar{\Omega})^{n}$ such that $h \cdot \nu \geq 0$ for all $x \in \Gamma, h=\nu$ on $\Gamma_{0}$ and $h=0$ on $\Omega \backslash \omega$ and let $\eta \in C^{2}([0, T])$ such that $\eta(0)=\eta(T)=0$ and $\eta(t)=1$ in $(\epsilon, T-\epsilon)$. We define $\theta(x, t)=\eta(t) h(x)$ wich belongs to $W^{2, \infty}(Q)$ and satisfies:

$$
\mid \begin{aligned}
& \theta(x, t)=\nu(x) \text { for all }(x, t) \in \Gamma_{0} \times(\epsilon, T-\epsilon), \\
& \theta(x, t) \cdot \nu(x) \geq 0, \text { for all }(x, t) \in \Gamma \times(0, T), \\
& \theta(x, 0)=\theta(x, T)=0, \text { for all } x \in \Omega, \\
& \theta(x, t)=0 \text { in }(\Omega \backslash \omega) \times(0, T) .
\end{aligned}
$$

Then, we consider the multiplier $\theta \cdot \nabla \phi$ and, as in the Lemma A.1, we obtain the following identity for all weak solution $\phi$ of (2.4):

$$
\begin{aligned}
\frac{1}{2} \iint_{\Sigma} \theta_{k}(x, t) \nu_{k}(x)\left(\frac{\partial \phi}{\partial \nu}\right)^{2} d \Sigma= & \left.\left(\phi^{\prime}(.), \theta(x, .) \cdot \nabla \phi(.)\right)\right|_{0} ^{T}+\iint_{Q} \frac{\partial \theta_{k}}{\partial x_{j}} \frac{\partial \phi^{i}}{\partial x_{k}} \frac{\partial \phi^{i}}{\partial x_{j}} d x d t \\
& +\frac{1}{2} \iint_{Q} \frac{\partial \theta_{k}}{\partial x_{k}}\left(\left|\phi^{\prime}\right|^{2}-|\nabla \phi|^{2}\right) d x d t+\iint_{Q} \frac{\partial q}{\partial x_{i}} \theta_{k} \frac{\partial \phi^{i}}{\partial x_{k}} d x d t .
\end{aligned}
$$

To $\theta$ as above we obtain:

$$
\frac{1}{2} \int_{\epsilon}^{T-\epsilon} \int_{\Gamma_{0}}\left(\frac{\partial \phi}{\partial \nu}\right)^{2} d \Sigma d t \leq \frac{1}{2} \iint_{\Sigma} \theta_{k}(x, t) \nu_{k}(x)\left(\frac{\partial \phi}{\partial \nu}\right)^{2} d \Sigma,
$$

since $\theta(x, t)=\nu(x)$ on $\Gamma_{0} \times(\epsilon, T-\epsilon)$ and

$$
\left.\left(\phi^{\prime}(.), \theta(x, .) \cdot \nabla \phi(.)\right)\right|_{0} ^{T}=0 .
$$

Since $\theta \in C^{1}(\bar{\Omega} \times(0, T))$, we have

$$
\left|\iint_{Q} \frac{\partial \theta_{k}}{\partial x_{j}} \frac{\partial \phi^{i}}{\partial x_{k}} \frac{\partial \phi^{i}}{\partial x_{j}} d x d t\right| \leq C \iint_{\omega \times(0, T)}|\nabla \phi|^{2} d x d t .
$$

For the pressure, we use Remark 3.2 to see that

$\iint_{Q} \frac{\partial q}{\partial x_{i}} \theta_{k} \frac{\partial \phi^{i}}{\partial x_{k}} d x d t=-\iint_{Q} \nabla q \phi \cdot \nabla \theta d x d t+\iint_{Q} \nabla q \phi(\operatorname{div} \theta) d x d t=\langle\nabla q,-\phi \cdot \nabla \theta+\phi(\operatorname{div} \theta)\rangle_{H^{-1}(Q), H_{0}^{1}(Q)}$ 
Consequently

$$
\left|\iint_{Q} \frac{\partial q}{\partial x_{i}} \theta_{k} \frac{\partial \phi^{i}}{\partial x_{k}} d x d t\right| \leq \delta\|\nabla q\|_{H^{-1}(Q)}^{2}+C_{\delta} \iint_{\omega \times(0, T)}\left(|\phi|^{2}+\left|\phi^{\prime}\right|^{2}+|\nabla \phi|^{2}\right) d x d t .
$$

Thus,

$$
\frac{1}{2} \iint_{\Sigma} \theta_{k}(x, t) \nu_{k}(x)\left(\frac{\partial \phi}{\partial \nu}\right)^{2} d \Sigma \leq C \iint_{\omega \times(0, T)}\left(|\phi|^{2}+\left|\phi^{\prime}\right|^{2}+|\nabla \phi|^{2}\right) d x d t+\delta\|\nabla q\|_{H^{-1}(Q)}^{2} .
$$

Using the fact that

$$
\|\nabla q\|_{H^{-1}(Q)}^{2} \leq C E(0),
$$

we conclude, choosing $\delta$ small enough, that

$$
E(0) \leq \hat{C} \int_{\epsilon}^{T-\epsilon} \int_{\Gamma_{0}}\left(\frac{\partial \phi}{\partial \nu}\right)^{2} d \Sigma \leq C \iint_{\omega \times(0, T)}\left(\left|\phi^{\prime}\right|^{2}+|\phi|^{2}+|\nabla \phi|^{2}\right) d x d t .
$$

Finally, by change of variables, we have

$$
E(0) \leq C \int_{\epsilon}^{T-\epsilon} \int_{\omega}\left(|\phi|^{2}+\left|\phi^{\prime}\right|^{2}+|\nabla \phi|^{2}\right) d x d t .
$$

Now let $\omega_{0}$ be a neighborhood of $\bar{\Gamma}_{0}$ such that $\Omega \cap \omega_{0} \subset \omega$. And we observe that the inequality (3.5) is true for each neighborhood of $\bar{\Gamma}_{0}$, the in particular for $\omega_{0}$, that is:

$$
E(0) \leq C \int_{\epsilon}^{T-\epsilon} \int_{\omega_{0}}\left(|\phi|^{2}+\left|\phi^{\prime}\right|^{2}+|\nabla \phi|^{2}\right) d x d t
$$

Now we consider $\rho \in W^{1, \infty}(\Omega), \rho \geq 0$ such that

$$
\rho=1 \text { in } \omega_{0} \text {, and } \rho=0 \text { in } \Omega \backslash \omega .
$$

We define $h(x, t) \in Q$ by $h(x, t)=\eta(t) \rho^{2}(x)$, where $\eta$ is defined above. We have

$$
\mid \begin{aligned}
& h(x, t)=1 \text { for all }(x, t) \in \omega_{0} \times(\epsilon, T-\epsilon), \\
& h(x, t)=0, \text { for all }(x, t) \in(\Omega \backslash \omega) \times(0, T), \\
& h(x, 0)=h(x, T)=0, \text { for all } x \in \Omega, \\
& \frac{|\nabla h|}{h} \in L^{\infty}(Q) .
\end{aligned}
$$

Multiplying both sides of $(2.4)_{1}$ by $h \phi$ and integrate by parts in $Q$, we obtain

$$
\iint_{Q} h \phi \cdot \phi^{\prime \prime} d x d t-\iint_{Q} h \phi \cdot \Delta \phi d x d t+\iint_{Q} h \nabla q \cdot \phi d x d t=0 .
$$

We have

$$
\int_{0}^{T} \int_{\Omega} \phi^{\prime \prime} h \phi d x d t=-\int_{0}^{T} \int_{\Omega} h\left|\phi^{\prime}\right|^{2} d t-\int_{0}^{T} \int_{\Omega} h^{\prime} \phi \phi^{\prime} d t .
$$

For the second, since $\phi=0$ on $\Sigma$, we have

$$
-\iint_{Q} h \Delta \phi \cdot \phi=\iint_{Q} \nabla \phi \nabla(h \phi) d x d t=\iint_{Q} h|\nabla \phi|^{2} d x d t+\iint_{Q} \phi \cdot(\nabla \phi \cdot \nabla h) d x d t .
$$


Consequently

$$
\iint_{Q} h|\nabla \phi|^{2} d t d t=\iint_{Q} h\left|\phi^{\prime}\right|^{2} d t+\iint_{Q} h^{\prime} \phi \cdot \phi^{\prime} d t-\iint_{Q} \phi \cdot(\nabla \phi \cdot \nabla h) d x d t-\iint_{Q} h \nabla q \cdot \phi d x d t
$$

It is immediate that

$$
\left|\iint_{Q} \phi \cdot(\nabla \phi \cdot \nabla h) d x d t\right| \leq \frac{1}{2} \iint_{Q} h|\nabla \phi|^{2} d x d t+\frac{1}{2} \iint_{Q} \frac{|\nabla h|^{2}}{h}|\phi|^{2} d x d t .
$$

Hence

$$
\iint_{Q} h|\nabla \phi|^{2} d t d t \leq C \iint_{\omega \times(0, T)}\left(\left|\phi^{\prime}\right|^{2}+|\phi|^{2}\right) d x d t+2\left|\iint_{Q} h \nabla q \cdot \phi d x d t\right| .
$$

Next, we observe that

$\iint_{Q} h \nabla q \cdot \phi d x d t=\langle q, \phi \cdot \nabla h\rangle_{H^{-1}\left(0, T ; L^{2}(\Omega)^{N}\right), H_{0}^{1}\left(0, T ; L^{2}(\Omega)^{N}\right)} \leq \delta\|q\|_{H^{-1}\left(0, T ; L^{2}(\Omega)^{N}\right)^{2}}^{2}+C_{\delta}\|h \phi\|_{H_{0}^{1}\left(0, T ; L^{2}(\Omega)^{N}\right)}^{2}$, for all $\delta>0$.

Therefore,

$$
\int_{\epsilon}^{T-\epsilon} \int_{\omega_{0}}|\nabla \phi|^{2} d x d t \leq C \iint_{\omega \times(0, T)}\left(\left|\phi^{\prime}\right|^{2}+|\phi|^{2}\right) d x d t+\delta\|q\|_{H^{-1}\left(0, T ; L^{2}(\Omega)^{N}\right)}^{2}
$$

which gives

$$
E(0) \leq C \iint_{\omega \times(0, T)}\left(\left|\phi^{\prime}\right|^{2}+|\phi|^{2}\right) d x d t+\delta\|q\|_{H^{-1}\left(0, T ; L^{2}(\Omega)^{N}\right)}^{2} .
$$

Finally, since $\|q\|_{H^{-1}\left(0, T ; L^{2}(\Omega)^{N}\right)}^{2} \leq C E(0)$, we obtain, for $\delta$ small enough,

$$
E(0) \leq C \iint_{\omega \times(0, T)}\left(\left|\phi^{\prime}\right|^{2}+|\phi|^{2}\right) d x d t .
$$

Using density arguments, we can prove that (3.6) holds for all weak solutions of (2.4).

\section{Internal OBservability InEQUALity}

This section is devoted to prove inequality (2.7). Indeed, we have:

Theorem 4.1. Assume $\omega$ satisfies (2.2) and let $T>2 R\left(x_{0}\right)$. Then, there exists a constant $C>0$ such that, for every $\left(\phi^{0}, \phi^{1}\right) \in V \times H$, the weak solution $\phi$ of (2.4) satisfies (2.7).

Proof. Let us suppose that (2.7) is not true. Then, given a natural number $n$, there exists an initial data $\left(\tilde{\phi_{n}^{0}}, \tilde{\phi}_{n}^{1}\right)$ such that $\tilde{\phi}_{n}$, the solution of $(2.4)$ corresponding to this initial data, satisfies

$$
\left\|\tilde{\phi_{n}^{0}}\right\|_{V}^{2}+\left|\tilde{\phi_{n}^{1}}\right|_{H}^{2} \geq n\left\|\tilde{\phi_{n}^{\prime}}\right\|_{\left.L^{2}(\omega \times(0, T))\right)} .
$$

We set

and

$$
K=\left(\left\|\tilde{\phi_{n}^{0}}\right\|_{V}^{2}+\left|\tilde{\phi_{n}^{1}}\right|_{H}^{2}\right)^{1 / 2}
$$

$$
\phi_{n}^{0}=\frac{\tilde{\phi}_{n}^{0}}{K}, \quad \phi_{n}^{1}=\frac{\tilde{\phi}_{n}^{1}}{K}, \quad \phi_{n}=\frac{\tilde{\phi_{n}}}{K} .
$$


We have

$$
\left\|\phi_{n}^{\prime}\right\|_{\left.L^{2}(\omega \times(0, T))\right)}^{2} \leq \frac{1}{n}
$$

and

$$
\left\|\phi_{n}^{0}\right\|_{V}^{2}+\left|\phi_{n}^{1}\right|_{H}^{2}=1
$$

From (4.1), there exist subsequences, denoted by the same index, such that

$$
\begin{gathered}
\liminf _{n \longrightarrow \infty} \iint_{\omega \times(0, T)}\left|\phi_{n}^{\prime}\right|^{2} d x d t=0, \\
\phi_{n}^{0} \rightarrow \phi^{0} \quad \text { in } V
\end{gathered}
$$

and

$$
\phi_{n}^{1} \rightarrow \phi^{1} \text { in } H
$$

Since $\phi_{N}$ is the solution of (2.4) associated to the initial data $\left(\phi_{n}^{0}, \phi_{n}^{1}\right)$, we have:

$$
\mid \begin{aligned}
& \phi_{n} \text { is bounded in } L^{\infty}(0, T ; V), \\
& \phi_{n}^{\prime} \text { is bounded in } L^{\infty}(0, T ; H) .
\end{aligned}
$$

Then, there exists a sebsequence $\phi_{N}$ such that

$$
\mid \begin{aligned}
& \phi_{n} \longrightarrow \phi \text { weak star in } L^{\infty}(0, T ; V) \\
& \phi_{n}^{\prime} \longrightarrow \phi^{\prime} \text { weak star in } L^{\infty}(0, T ; H) .
\end{aligned}
$$

From (4.7), it is not difficult to show that $\phi$ is the weak solution of (2.4) corresponding to the initial data $\left(\phi^{0}, \phi^{1}\right)$.

Next, since $V \hookrightarrow H$ compactly, estimate (4.7) and the Aubin-Lions compactness theorem give us

Hence, it follows from (4.3) and 4.7 that

$$
\phi_{n} \longrightarrow \phi \text { in } L^{2}(0, T ; H) .
$$

$$
\phi^{\prime} \equiv 0 \text { in } \omega \times(0, T)
$$

and $\phi$ is independent of $t$ in $\omega$.

Let us now consider the system

$$
\mid \begin{array}{ll}
\xi^{\prime \prime}-\Delta \xi+\nabla q=0 & \text { in } Q, \\
\operatorname{div} \xi=0 & \text { in } Q, \\
\xi=0 & \text { on } \Sigma, \\
\xi(0)=\phi^{1} \in H, \xi^{\prime}(0)=\Delta \phi^{0} \in W . &
\end{array}
$$

Taking $\psi(x, t)=\phi^{0}(x)+\int_{0}^{t} \xi(x, s) d s$, it is not difficult to see that $\psi$ solves $(2.4)$, with $\left(\phi^{0}, \phi^{1}\right)$ as initial data. Therefore, from the uniqueness of solutions to (2.4), we have that $\psi \equiv \phi$ and thanks to (4.9) we have that $\xi \equiv 0$ in $\omega \times(0, T)$.

Let us now show that $\xi \equiv 0$. Applying the curl operator in (4.10), we see that $v=\operatorname{curl} \xi$ satisfies

$$
\mid \begin{array}{ll}
v^{\prime \prime}-\Delta v=0 & \text { in } Q \\
v \equiv 0 & \text { in } \omega \times(0, T)
\end{array}
$$


Then, by Holmgren's Uniqueness Theorem (see [19]), we deduce that $v \equiv 0$. Therefore, there exists a scalar function $\Phi=\Phi(x, t)$ such that

$$
\xi=\nabla \Phi \text { in } Q
$$

In view of $(4.10)_{2}$, we have

$$
\Delta \Phi=0 \text { in } Q
$$

Since $\xi=0$ in $\omega \times(0, T)$, we also have

$$
\Phi=f(t) \text { in } \omega \times(0, T) .
$$

and from the unique continuation for the Laplace equation, we deduce that

$$
\Phi=f(t) \text { in } Q
$$

Hence, we see that

$$
\xi=\nabla \Phi=0 \text { in } Q .
$$

As a consequence of (4.12), we obtain

$$
\phi^{1}=\phi^{0}=0 .
$$

From (3.1), (4.8) and (4.13), we get a contradiction, and the proof is finished.

Since we have shown that the observability inequality (2.5) holds for all solutions of (2.4), we are able to prove the internal null controllability for the hyperbolic system with a pressure term (2.3).

Theorem 4.2. Assume $\omega$ satisfies (2.2) and let $T>2 R\left(x_{0}\right)$. Given $\left(u_{0}, u_{1}\right) \in V \times H$, there exists a control $h \in L^{2}(\omega \times(0, T))$ such that the associated solution $u$ of (2.3) satisfies

$$
u(T)=u^{\prime}(T)=0 .
$$

Moreover, there exists a positive constant $C$, such that the following holds

$$
\iint_{\omega \times(0, T)}|h|^{2} d x d t \leq C\left(\left\|u_{0}\right\|_{V}^{2}+\left|u^{1}\right|_{H}^{2}\right) .
$$

Proof. Since the proof is similar to the one for the wave equation, we will just give a sketch of the proof.

We consider the functional

$$
\mathcal{J}: H \times W \longrightarrow \mathbb{R}
$$

given by

$$
\mathcal{J}\left(\phi^{0}, \phi^{1}\right)=\frac{1}{2} \iint_{\omega \times(0, T)}|\phi|^{2} d x d t+<\varphi^{1}, u^{0}>_{H^{-1}(\Omega), H_{0}^{1}(\Omega)}-\left(\phi^{0}, u^{1}\right)_{H},
$$

where $\varphi$ is the solution of (2.4) corresponding to the initial data $\left(\phi^{0}, \phi^{1}\right)$.

Using the observability inequality (2.5) and energy estimates, we can show that the functional $\mathcal{J}$ is continuous, strictly convex and coercive. Therefore, the $\mathcal{J}$ has a unique minimizer $\left(\hat{\phi}^{0}, \hat{\phi}^{1}\right)$. Using the Euler-Lagrange equation of the functional, we conclude that $\hat{\phi}$, the solution of (2.4) associated to $\left(\hat{\phi}^{0}, \hat{\phi}^{1}\right)$, is a control which drives $u$ to zero in time $T$. Inequality (4.14) then follows from the observability inequality (2.5) and the fact that $\mathcal{J}\left(\hat{\phi}^{0}, \hat{\phi}^{1}\right) \leq 0$. This finishes the proof of Theorem 4.2. 


\section{The Stokes System With Regular Data}

In this section we prove that the Stokes system with regular initial data is null controllable with a cost of order $e^{C / T}$. For this, we will use the Control Transmutation Method and the results of the previous section.

In order to make things clear, we introduce two different time intervals $(0, T)$ and $(0, L)$ and consider the Stokes system

$$
\mid \begin{array}{ll}
y_{t}-\Delta y+\nabla p=g 1_{\omega} & \text { in } Q_{t}:=\Omega \times(0, T), \\
\operatorname{div} y=0 & \text { in } Q_{t}, \\
y=0 & \text { on } \Sigma_{t}:=\partial \Omega \times(0, T), \\
y(0)=y_{0} & \text { in } \Omega
\end{array}
$$

and the associated hyperbolic system with a pressure term

$$
\mid \begin{array}{ll}
u_{l l}-\Delta u+\nabla q=h 1_{\omega} & \text { in } Q_{l}:=\Omega \times(0, L), \\
\text { div } u=0 & \text { in } Q_{l}, \\
u=0 & \text { on } \Sigma_{l}:=\partial \Omega \times(0, L), \\
u(0)=y_{0}, u^{\prime}(0)=0 & \text { in } \Omega
\end{array}
$$

in $\Omega \times(0, T)$ and $\Omega \times(0, L)$, respectively.

We take $y_{0} \in V, L>2 R\left(x_{0}\right), \Omega$ and $\omega$ as in section 2. It follows from Theorem 4.2 that system (5.2) is null controllable with a control $h \in L^{2}(\omega \times(0, L))$ satisfying (4.14).

For what follows, the following theorem will be very useful.

Theorem 5.1. There exists a positive constant $\alpha^{*}$ such that, for all $\alpha>\alpha^{*}$, there exists $\gamma>0$ having the property that, for all $L>0$ and $T \in\left(0, \inf (\pi / 2, L)^{2}\right]$, there exists a distribution $k \in C([0, T] ; \mathcal{M}(-L, L)))$ satisfying

$$
\mid \begin{aligned}
& k_{t}=\partial_{s}^{2} k \text { in } \mathcal{D}^{\prime}((0, T) \times(-L, L)) \\
& k(0, x)=\delta(0) \\
& k(T, x)=0 \\
& \|k\|_{L^{2}((0, T) \times(-L, L))}^{2} \leq \gamma e^{\alpha L^{2} / T}
\end{aligned}
$$

Proof. See [21].

We have:

Theorem 5.2. Assume $\omega$ satisfies (2.2), $y_{0} \in V$ and $T>0$. Then, there exists a control $g \in L^{2}(\omega \times(0, T)$ such that the solution $y$ of (5.1) satisfies

$$
y(T)=0
$$

and

for a constant $C>0$.

$$
\iint_{\omega \times(0, T)}|y|^{2} d x d t \leq C \gamma e^{\alpha L^{2} / T}\left\|y_{0}\right\|_{V}^{2}
$$


Proof. We start the proof extending $k$ by zero outside $[0, T] \times(-L, L)$ and extend $u$ and $h$ by reflection to $[-L, 0]$ and by zero outside $[-L, L]$.

We set

$$
y(t)=\int k(t, s) u(s) d s
$$

and

$$
g(t)=\int k(t, s) h(s) d s .
$$

We must prove that $(y, g)$ solves, together with some $p$, the stokes system (5.1). Indeed, the following two properties are easy to check

$$
\text { divy }=0 \text { in } Q \text { and } y=0 \text { on } \Sigma .
$$

Next, taking $\varphi \in V$, we have

wich implies

$$
<y(t), \varphi>_{\mathcal{D}^{\prime}(\Omega), \mathcal{D}(\Omega)}=<\int k(t, s) u(s) d s, \varphi>_{\mathcal{D}^{\prime}(\Omega), \mathcal{D}(\Omega)},
$$

$$
<y_{t}(t), \varphi>_{\mathcal{D}^{\prime}(\Omega), \mathcal{D}(\Omega)}=<\int k_{t}(t, s) u(s) d s, \varphi>_{\mathcal{D}^{\prime}(\Omega), \mathcal{D}(\Omega)} .
$$

Using the properties of $k$, we see that

$$
<y_{t}(t), \varphi>_{\mathcal{D}^{\prime}(\Omega), \mathcal{D}(\Omega)}=<\int k_{s s}(t, s) u(s) d s, \varphi>_{\mathcal{D}^{\prime}(\Omega), \mathcal{D}(\Omega)} .
$$

Integrating by parts, and using the fact that $u(-L)=u(L)=u^{\prime}(-L)=u^{\prime}(L)=0$, we obtain

$$
<y_{t}(t), \varphi>_{\mathcal{D}^{\prime}(\Omega), \mathcal{D}(\Omega)}=<\int k(t, s) u_{s s}(s) d s, \varphi>_{\mathcal{D}^{\prime}(\Omega), \mathcal{D}(\Omega)},
$$

i.e.,

$$
<y_{t}(t), \varphi>_{\mathcal{D}^{\prime}(\Omega), \mathcal{D}(\Omega)}=\int k(t, s)<u_{s s}(s), \varphi>_{\mathcal{D}^{\prime}(\Omega), \mathcal{D}(\Omega)} d s .
$$

Since $u$ is, together with some $q$, the solution of (5.2), we have

$$
<y_{t}(t), \varphi>_{\mathcal{D}^{\prime}(\Omega), \mathcal{D}(\Omega)}=\int k(t, s)<\Delta u(s)+h 1_{\omega}, \varphi>_{\mathcal{D}^{\prime}(\Omega), \mathcal{D}(\Omega)} d s .
$$

Therefore,

$$
<y_{t}(t), \varphi>_{V^{\prime}, V}=<\int k(t, s) \Delta u(s) d s, \varphi>_{\mathcal{D}^{\prime}(\Omega), \mathcal{D}(\Omega)}+<\int k(t, s) h 1_{\omega} d s, \varphi>_{\mathcal{D}^{\prime}(\Omega), \mathcal{D}(\Omega)} .
$$

This last identity gives

$$
<y_{t}(t)-\Delta y(t), \varphi>_{\mathcal{D}^{\prime}(\Omega), \mathcal{D}(\Omega)}=<g(t) 1_{\omega}, \varphi>_{\mathcal{D}^{\prime}(\Omega), \mathcal{D}(\Omega)} .
$$

Since $k$ is a controlled solution of the heat equation, with a Dirac mass at the origin, we conclude that $y(0)=y_{0}$ and $y(T)=0$.

Therefore, we have shown the existence of control $g$ such that the solution $y$ of (5.1), together with some $p$, associated to $y_{0}$ is driven to rest at time $T$. 
Let us now prove estimate (6.1). From the transmutation formula for the control $g$, it is immediate that

$$
\iint_{\omega \times(0, T)}|g|^{2} d x d t \leq\|k\|_{L^{2}((0, T) \times(-L, L))}^{2}\|h\|_{L^{2}(\omega \times(0, T))}^{2} .
$$

Finally, using the properties of $k$ and Theorem 4.2, inequality (6.1) follows.

\section{The Stokes System With Less Regular Data}

In this section we improve the result obtained in the previous section. We prove that we can take less regular initial data and still have null controllability with a cost of order $e^{C / T}$. In order to show the result, we combine Theorem 5.2, energy inequalities and the smoothing effect for the Stokes system.

The result is as follows.

Theorem 6.1. Assume $\omega$ satisfies (2.2), $y_{0} \in H, T>0$ and $0<\epsilon<T$. Then, there exists a control $g \in L^{2}(\omega \times(0, T)$ such that the solution $y$ of (5.1) satisfies

$$
y(T)=0
$$

and

$$
\iint_{\omega \times(0, T)}|y|^{2} d x d t \leq \epsilon \gamma C e^{\frac{2}{\epsilon}} e^{\alpha L^{2} / T}\left|y_{0}\right|_{H}^{2}
$$

for a constant $C>0$.

Proof. Fist, we let system (5.1) evolve freely in the interval $(0, \epsilon)$. Using the smoothing effect of the Stokes system we have that $y(\epsilon)=y_{\epsilon}$ belongs to $V$. We also have, thanks to Theorem 5.2, that there exists $g \in L^{2}(\omega \times(0, T-\epsilon))$ such that the associated solution $y$ to the problem

$$
\mid \begin{array}{ll}
y_{t}-\Delta y+\nabla p=g \chi_{\omega} & \text { in }(0, T-\epsilon) \times \Omega, \\
\operatorname{div} y=0 & \text { in }(0, T-\epsilon) \times \Omega, \\
y=0 & \text { on }(0, T-\epsilon) \times \partial \Omega, \\
y(0)=y_{\epsilon} & \text { in } \Omega,
\end{array}
$$

satisfies:

$$
y(T-\epsilon)=0
$$

Moreover,

$$
\int_{0}^{T-\epsilon} \int_{\omega}|g|^{2} d x d t \leq C \gamma e^{\alpha L^{2} / T}|| y_{\epsilon} \|_{V}^{2}
$$

Let us now define functions $\bar{y}$ and $\bar{g}$ by $\bar{y}(t+\epsilon)=y(t), \bar{g}(t+\epsilon)=g(t)$ for $0<t<T-\epsilon$. The functions $\bar{y}$ and $\bar{g}$ are defined in $(\epsilon, T)$ and satisfy

$$
\begin{array}{ll}
\bar{y}_{t}-\Delta \bar{y}+\nabla \bar{q}=\bar{g} \chi_{\omega} & \text { in }(\epsilon, T) \times \Omega, \\
\text { div } \bar{y}=0 & \text { in }(\epsilon, T) \times \Omega, \\
\bar{y}=0 & \text { on }(\epsilon, T) \times \partial \Omega, \\
\bar{y}(\epsilon)=y_{\epsilon} & \text { in } \Omega
\end{array}
$$


Inequality (6.3) then becomes

$$
\int_{\epsilon}^{T} \int_{\omega}|\bar{g}|^{2} d x d t \leq C \gamma e^{\alpha L^{2} / T}\left\|y_{\epsilon}\right\|_{V}^{2}
$$

Next, we set

$$
g(t)=\left\{\begin{aligned}
0 ; & \text { if } \quad 0<t<\epsilon \\
\bar{g}(t) ; & \text { if } \quad \epsilon \leq t<T
\end{aligned}\right.
$$

It is not difficult to see that the solution $y$ to (5.1), with $g$ as a control, satisfies $y(T)=0$. From (6.5), and the definition of $g$, we have the following estimate, which we call "pseudo-cost" of the controllability:

$$
\int_{0}^{T} \int_{\omega}|y|^{2} d x d t \leq C \gamma e^{\alpha L^{2} / T}|| y_{\epsilon} \|_{V}^{2}
$$

Let us now consider system $(5.1)$ in the interval $[0, \epsilon]$, i.e., we consider the system

with $y_{0} \in H$.

$$
\begin{array}{ll}
y_{t}-\Delta y+\nabla p=0 & \text { in }(0, \epsilon) \times \Omega, \\
\text { div } y=0 & \text { in }(0, \epsilon) \times \Omega, \\
y=0 & \text { on }(0, \epsilon) \times \partial \Omega, \\
y(0)=y_{0} & \text { in } \Omega,
\end{array}
$$

We make the change of variable $z(t)=e^{-\frac{1}{t}} y(t)$. This new function $z$ solves

$$
\mid \begin{array}{ll}
z_{t}-\Delta z+\nabla p=\frac{1}{t^{2}} e^{-\frac{1}{t}} y & \text { in }(0, \epsilon) \times \Omega, \\
\operatorname{div} z=0 & \text { in }(0, \epsilon) \times \Omega, \\
z=0 & \text { on }(0, \epsilon) \times \Sigma, \\
u(0)=0 & \text { in } \Omega .
\end{array}
$$

Using the fact that $\frac{1}{t^{2}} e^{-\frac{1}{t}} y \in L^{2}(0, \epsilon ; H)$ and the regularity of the Stokes system, we conclude that $z \in L^{2}\left(0, \epsilon ; H^{2}(\Omega)\right)$ and $z_{t} \in L^{2}(0, \epsilon ; H)$.

Multiplying (6.8) by $z_{t}$ and integrating by parts, we get

$$
2\left|z_{t}(t)\right|_{H}^{2}+\frac{d}{d t}\|z(t)\|_{V}^{2}=2\left(\frac{1}{t^{2}} e^{-\frac{1}{t}} y(t), z_{t}\right)_{H} .
$$

Integrating (6.9) from 0 to $\epsilon$ and using Young's inequality, we obtain

$$
\int_{0}^{\epsilon}\left|z_{t}(t)\right|_{H}^{2} d t+\|\left. z(\epsilon)\right|_{V} ^{2} \leq C \int_{0}^{\epsilon}\left|\frac{1}{t^{2}} e^{-\frac{1}{t}} y(t)\right|_{H}^{2} d t+\delta \int_{0}^{\epsilon}\left|z_{t}\right|_{H}^{2} d t,
$$

for all $\delta>0$.

Taking $\delta$ small enough, we have

$$
\|z(\epsilon)\|_{V}^{2} \leq C \int_{0}^{\epsilon}\left|\frac{1}{t^{2}} e^{-\frac{1}{t}} y(t)\right|_{H}^{2} d t
$$

and, since for $\epsilon$ sufficiently small, we have $\frac{1}{t^{4}} e^{-\frac{2}{t}} \leq e^{\frac{1}{\epsilon}}$ for $0<t<\epsilon$, it follows that

$$
\|z(\epsilon)\|_{V}^{2} \leq e^{\frac{1}{\epsilon}} \int_{0}^{\epsilon}|y(t)|_{H}^{2} d t
$$


Finally, using the fact that $\|\left. y\right|_{L^{2}(0, \epsilon ; H)} ^{2} \leq \epsilon\left|y_{0}\right|_{H}^{2}$, we get from (6.10) that

$$
\|z(\epsilon)\|_{V}^{2} \leq \epsilon e^{\frac{1}{\epsilon}}\left|y_{0}\right|_{H}^{2}
$$

and, in particular, using the fact that $z(t)=e^{-\frac{1}{t}} y(t)$, we conclude that

$$
\|y(\epsilon)\|_{V}^{2} \leq \epsilon e^{\frac{2}{\epsilon}}\left|y_{0}\right|_{H}^{2}
$$

From (6.6) and (6.11), the result then follows.

Remark 6.2. Since $y_{\epsilon} \longrightarrow y_{0}$ in $H$, the norm of $y_{\epsilon}$ is not bounded in $V$. Hence, we can not pass to the limit in (6.6) when $\epsilon \longrightarrow 0$.

\section{Appendix A. Some AuXiLiary Lemmas}

In what concerns the proof of Theorem 2.3, the following Lemmas are in order.

Lemma A.1. Let $\bar{q}=\bar{q}(x)$ be in $C^{1}(\bar{\Omega})^{n}$, then for every regular solution u of (2.3), the following identity holds:

$$
\begin{aligned}
\frac{1}{2} \iint_{\Sigma} \bar{q}_{k}(x) \nu_{k}(x)\left(\frac{\partial u}{\partial \nu}\right)^{2} d \Sigma= & \left.\left(u^{\prime}(t), \bar{q}(x) \nabla u(t)\right)\right|_{0} ^{T}+\iint_{Q} \frac{\partial \bar{q}_{k}}{\partial x_{j}} \frac{\partial u^{i}}{\partial x_{k}} \frac{\partial u^{i}}{\partial x_{j}} d x d t \\
& +\frac{1}{2} \iint_{Q} \frac{\partial \bar{q}_{k}}{\partial x_{k}}\left(\left|u^{\prime}\right|^{2}-|\nabla u|^{2}\right) d x d t \\
& +\iint_{Q} \frac{\partial p}{\partial x_{i}} \bar{q}_{k} \frac{\partial u^{i}}{\partial x_{k}} d x d t+\iint_{Q} h^{i} \bar{q}_{k} \frac{\partial u^{i}}{\partial x_{k}} d x d t
\end{aligned}
$$

Proof. The proof is the same as in the case of a single wave equation, the difference is that here see the pressure as a force term in the right-hand side.

Lemma A.2. Let $\nu=\left(\nu_{1}, \ldots, \nu_{n}\right)$ the vector field of exterior normal to $\partial \Omega$. Then, there exists a vector field $k=\left(k_{1} \ldots, k_{n}\right) \in C^{1}(\bar{\Omega})^{n}$ such that

$$
k_{i}=\nu_{i} \text { on } \partial \Omega \text { for } i=1, \ldots, n \text {. }
$$

Proof. We consider the trace bijection operator $\gamma_{0}: H^{m}(\Omega) \rightarrow H^{m-\frac{1}{2}}(\partial \Omega)$. Then, if $\nu_{j} \in$ $H^{m-\frac{1}{2}}(\partial \Omega)$, there exists $k_{j} \in H^{m}(\Omega)$ such that $\gamma_{0} k_{j}=\nu_{j}$. From Sobolev's embedding theorem, we know that for $m>1+\frac{n}{2}$ we have $H^{m}(\Omega) \subset C^{1}(\bar{\Omega})$ continuously, and the results follows.

Lemma A.3. Let $\left(u^{0}, u^{1}, h\right) \in V \times H \times L^{2}(0, T ; H)$, then the weak solution of (2.3) satisfies:

$$
\iint_{\Sigma}\left(\frac{\partial u}{\partial \nu}\right)^{2} d \Sigma \leq C\left(\left|u^{1}\right|_{H}^{2}+\left\|u^{0}\right\|_{V}^{2}+\|h\|_{L^{2}(0, T ; H)}^{2}\right)
$$

Proof. The proof is performed as the equivalent one for the wave equation, first showing the result for regular solutions. Indeed, in this case we must take the vector field $\bar{q}$ in Lemma A.1 to be the vector field $\bar{q}(x)=x$ and use the fact that

$$
\iint_{Q} \frac{\partial p}{\partial x_{i}} \bar{q}_{k} \frac{\partial u^{i}}{\partial x_{k}} d x d t=0
$$




\section{REFERENCES}

[1] G. O. Antunes, R. S. Busse, H. R. Cripa, Hidden regularity for a nonlinear hyperbolic equation with a resistance Term, International Mathematical Forum, 4 (11)(2009), 511-520.

[2] G. O. Antunes, F. D. Araruna, L. A. Medeiros, Simultaneous controllability for a system with resistance term, Tendncias em Matemática Aplicada e Computacional, 3 (1)(2002), 31-40.

[3] M. M. Cavalcanti, V. N. Domingos Cavalcanti, A. Rocha, J. A. Soriano, Exact Controllability of a secondorder integro-differential equation with a pressure term, EJQTDE, 9 (1998), 1-18.

[4] C. Fabre, Uniquiness results for Stokes equations and their consequences in linear and nonlinear control problems, ESAIM Control Optim. Calc. Var. 1, (1995/1996), 35-75.

[5] C. Fabre, J.-P. Puel, E. Zuazua, Approximated controllability of the semilinear heat equations, Proc. Roy. Soc. Edinburgh Sect. A, 125 (1995), 31-61.

[6] S. Ervedoza, E. Zuazua, Sharp observability estimates for heat equations, Arch. Rational Mech. Anal., 202 (3) (2011), 975-1017.

[7] S. Ervedoza, E. Zuazua, Observability of heat processes by transmutation without geometric restrictions, MCRF, 1 (2)(2011), 177-187.

[8] H. O. Fattorini, D. L. Russell. Exact controllability theorems for linnear parabolic equations in one space dimension, Arch. Rational Mech. Anal., 43 (1971), 272-292.

[9] E. Fernádez-Cara, S. Guerrero, O. Yu. Ymanuvilov, J.-P. Puel, Local exact controllability of the Navier-Stokes system, J. Math. Pures Appl., 83 (12)(2004), 1501-1542.

[10] E. Fernádez-Cara, S. Guerrero, Global Carleman Inequalities for Parabolic Systems and Applications to Controllability, SIAM J. Control and Optimization, 45 (4), 1395-1446.

[11] E. Fernández-Cara and E. Zuazua, The cost of approximate controllability for heat equations: the linear case, Adv. Diff. Equations, 5 (2000), 465-514.

[12] E. Fernández-Cara, E. Zuazua, Null and approximate controllability for weakly blowing up semilinear heat equations Annales de l'IHP. Analyse non linéaire, 17 (5) (2000), 583-616.

[13] A. V. Fursikov, O. Yu. Imanuvilov, Controllability of Evolutions Equations, Lectures Notes Series, Vol. 34, Seoul National University, 1996.

[14] S. Guerrero, Some recent controllability results for the Three-Dimensional Stokes system with two scalar controls, Bol. Soc. Esp. Mat. Aplic., 48 (2009), 7-29.

[15] S. Guerrero, Controllability of systems of Stokes equations with one control force: existence of insensitizing controls, Annales de l'Institut Henri Poincaré Analyse Non Linéaire, 24 (2007), no. 6, 1029-1054.

[16] R. B. Guenther, E. A. Thomann, Fundamental Solutions of Stokes and Oseen Problem in Two Spatial Dimensions, J. math. fluid mech., 9 (4)(2007), 489-505

[17] R. B. Guenther, E. A. Thomann, The Fundamental Solution of the Linearized Navier-Stokes Equations for Spinning Bodies in Three Spatial Dimensions Time Dependent Case, J. math. fluid mech., 8 (1)(2006), $77-98$.

[18] G. Lebeau, L. Robbiano, Controle exact de l'équation de la chaleur, Comm. Partial Differential Equations 20 (1995), 335-356.

[19] J.-L. Lions, Contrlabilité exacte perturbations et stabilisation de systémes distribués. Tome I. Contrlabilit Exacte, Rech. Math. Appl. 8, Masson, Paris, 1988.

[20] J.-L. Lions, On some Hyperbolic Equations with a Pressure term, Proceedings of the conference dedicated to Louis Nirenberg, Trento-Italy, September 3-8, 1990. Harlow: Longman Scientific and Technical Pitman Res. Notes Math. Ser., 269 (1992), 196-208.

[21] L. Miller, The Control Transmutation Method and the cost of fast controls, SIAM J. Control and Optimization, $45(2)(2006), 762-772$.

[22] L. Miller, Geometric bounds on the growth rate of null-controllability cost for the heat equation in small time, J. Differential Equations, 204 (1)(2004), 202-226.

[23] L. A. Medeiros, Exact Controllability for Wave Equations-HUM, 37 Seminrio Brasileiro de Anlise, Rio de Janeiro (1993), 63-173. 
[24] A. Rocha dos Santos, Exact controllability in dynamic incompressible materials. Ph.D. Thesis, Instituto de Matemática-UFRJ, Rio de Janeiro-Rj-Brasil, 1996.

[25] V. A. Solonnikov, Estimates for solutions of a non-stationary linearized system of NavierStokes equations, Trudy Mat. Inst. Steklov., 70 (1964), 213-317.

[26] R. Temam, Navier-Stokes Equations. Theory and Numerical Analysis, Stud. Math. Appl., vol. 2, NorthHolland, Amsterdam-New York-Oxford, 1977.

[27] G. Tenenbaum, M. Tucsnak, New blow-up rates for fast controls of Schrödinger and heat equations, J. Differential Equations, 243 (1)(2007), 70-100.

[28] E. Zuazua, Controllability of the linear system of thermoelasticity, J. Math. Pures App., 74 (1995), $291-315$.

BCAM - Basque Center for Applied Mathematics Mazarredo 14, 48009 Bilbao, Basque Country, SPAIN

E-mail address: chaves@bcamath.org 